\title{
When language comprehension reflects production constraints: Resolving ambiguities with the help of past experience
}

\author{
Maryellen C. MacDonald \\ University of Wisconsin-Madison, Madison, Wisconsin \\ AND \\ ROBERT THORNTON \\ Pomona College, Claremont, California
}

\begin{abstract}
A key assumption in language comprehension is that biases in behavioral data, such as the tendency to interpret John said that Mary left yesterday to mean that yesterday modifies the syntactically local verb left, not the distant verb said, reflect inherent biases in the language comprehension system. In the present article, an alternative production-distribution-comprehension (PDC) account is pursued; this account states that comprehension biases emerge from different interpretation frequencies in the language, which themselves emerge from pressures on the language production system to produce some structures more than others. In two corpus analyses and two self-paced reading experiments, we investigated these claims for verb modification ambiguities, for which phrase length is hypothesized to shape production. The results support claims that tendencies to produce short phrases before long ones create distributional regularities for modification ambiguities in the language and that learning over these regularities shapes comprehenders' interpretations of modification ambiguities. Implications for the PDC and other accounts are discussed.
\end{abstract}

English speakers who hear or read "Tom said that his cousins left yesterday" almost overwhelmingly interpret this sentence to mean that the cousins left yesterday, not that Tom reported this information yesterday. That is, people interpret the syntactically ambiguous adverbial yesterday to modify the more local verb left rather than the more distant verb said. This interpretation bias toward local modification is extremely strong in these verb modification ambiguities. In contrast to many other syntactic ambiguities, whose interpretation is readily affected by the precise words in the sentence (for a review, see MacDonald \& Seidenberg, 2006), the local modification preference in verb modification ambiguities is relatively immune to changes in lexical or discourse context, with a few fairly narrow exceptions (Altmann, van Nice, Garnham, \& Henstra, 1998; Fodor \& Inoue, 1994). One interpretation of this robust local modification preference is that it emerges from basic properties of the language comprehension system - essentially, that people resolve the ambiguity in favor of local modification because something about the parsing system produces this bias. Variants of this position include right association (Kimball, 1973), late closure (Frazier, 1987), and recency (Pearlmutter \& Gibson, 2001). The exact hypothesis varies across these positions, but these accounts all hold that the preference for local modification can be traced to some feature of the comprehension architecture that results in its computing the local modification interpretation first and considering the distant modification interpretation only if plausibility forces a reevaluation of the initial parse.

An alternative view of the local modification preference, consistent with constraint-based accounts of ambiguity resolution (MacDonald \& Seidenberg, 2006; Tanenhaus \& Trueswell, 1995), is that it does not emerge from an inherent bias in the language comprehension system, but from the constraint satisfaction process, because it is favored by probabilistic cues in the input. Relevant constraints have not been explicitly specified for this ambiguity, but discussions of similar ambiguities offer some likely possibilities. For example, Thornton, MacDonald, and Gil (1999) investigated sentences such as 1a and 1b, in which both the distant and the local modification sites were noun phrases (NPs), and found that the "modifiability" of the second NP influenced interpretation of a modifying prepositional phrase (PP).

1a. The puppy by a truck with floppy ears ...

1b. The puppy by Jim's truck with floppy ears ...

In sentences of this type, when the second NP was vague and could easily be described further, as with $a$ truck in 1a, comprehenders tended to interpret the following PP as modifying this second NP, and they exhibited long reading times when the meaning of this phrase (with floppy ears)

M. C. MacDonald, mcmacdonald@wisc.edu 
turned out to modify the first NP (the puppy). However, when the second NP was highly specific, such as Jim's truck in $1 \mathrm{~b}$, comprehenders readily interpreted the PP as modifying the distant NP. These results built upon work by Spivey-Knowlton and Sedivy (1995), who investigated modification ambiguities with one verb and one NP modification site and found that modifiability of both the NP and the verb affected interpretation.

Given these results, it seems plausible that relative modifiability of two verb modification sites could also play a role in verb modification ambiguities. For example, in Tom said that his cousins left yesterday, a leaving event seems to be a more plausible candidate for being modified with a temporal adverbial than a saying event is, so the relative preference for local modification might be driven by greater modifiability of left than of said. This might be true for these particular verbs, but one of the hallmarks of verb modification ambiguity is that the local modification preference is quite resistant to lexical changes; for example, Tom said that his cousins spoke yesterday also yields a preference for the local modification interpretation, despite the fact that there seems to be no obvious difference between the modifiability of said and that of spoke. Other constraints may also contribute to the local modification bias, but constraints that are not grounded in lexical or discourse properties have rarely been posited within constraint-based accounts, and it is not immediately obvious how to formulate them. A constraint that directly promotes local modification could turn out to be very similar to the architecturally based parsing principles that constraint-based accounts are designed to challenge. Thus, what is needed for a constraint-based account of this ambiguity is a formulation of a nonlexical (or not strongly lexical) constraint that is consistent with the probabilistic accounts of sentence comprehension that have been developed for other ambiguities (MacDonald \& Seidenberg, 2006; Tanenhaus \& Trueswell, 1995). A formulation of a constraint of this sort has importance beyond an account of this specific syntactic ambiguity because an introduction of nonlexical constraints is a significant extension for constraint-based accounts, which have been largely dominated by lexical-level biases, to the point that the approach is often called a constraint-based lexicalist account (see, e.g., Trueswell, 1996).

We offer a constraint of this sort together with an explanation for why a nonlexical constraint could come to dominate ambiguity resolution to such a large degree in verb modification ambiguities. Our approach is rooted in MacDonald's (Gennari \& MacDonald, 2009; MacDonald, 1999) production-distribution-comprehension (PDC) account, which argues that a major force shaping the distributional patterns of sentence structures in a language user's input are constraints on the language production system, which promotes certain structures and lexical/structural pairings over others. These production pressures, over time and across many speakers and writers, create distributional patterns in the language input that comprehenders perceive. Comprehenders implicitly learn from these patterns and interpret new input as consistent with previous experience. The PDC account has been tested extensively in accounts of relative clause comprehension with stud- ies of producers' syntactic and lexical choices (Gennari \& MacDonald, 2009; Race \& MacDonald, 2003), studies of implicit learning of distributional patterns (Wells, Christiansen, Race, Acheson, \& MacDonald, 2009), and studies linking reading times (a common measure of comprehension difficulty) to the availability of alternative interpretations and production patterns in the language (Gennari \& MacDonald, 2008, 2009). MacDonald and colleagues have suggested that the PDC account offers an alternative view of relative clause interpretation in which patterns of comprehension difficulty are not seen as reflecting architectural properties of the comprehension system but are instead attributed to statistical learning of distributional patterns in the input, which are themselves traced to pressures on the language production system.

A PDC account may similarly be applied to verb modification ambiguities. MacDonald (1999) first argued that production constraints shape the distribution of modification ambiguities, suggesting that the effects emerge from the relative length of different phrases in the sentence. Length is a well-known contributor to production choices; in English and in many other languages, there is a strong tendency for short phrases to precede long ones (Hawkins, 1994; Stallings, MacDonald, \& O'Seaghdha, 1998; Wasow, 1997). Among production researchers, this tendency is thought to emerge from the fact that language production is driven by accessibility: When there is an option in ordering, the order of words and phrases in a sentence is affected by which ones are ready to be produced (Bock, 1987; V. S. Ferreira, 1996; Kempen \& Hoenkamp, 1987). Short and/or less complex (Wasow \& Arnold, 2003) phrases require less production effort than longer, more complex ones do (F. Ferreira, 1991; Wheeldon \& Lahiri, 1997) and are consequently more likely to appear in earlier sentence positions (De Smedt, 1994). The placement of highly accessible phrases early in the sentence allows the speaker to begin speaking these easily planned items while continuing to plan the upcoming, more difficult material. In contrast, if the more difficult material is placed first, utterance initiation has to wait until this material is fully planned, and the production system has to maintain activation for rapidly planned, easier phrases until articulation processes get through sufficient parts of the sentence so that these sections can be uttered.

These accessibility-based production pressures are relevant to the distribution of sentences with modification ambiguities. In Tom said that his cousins left yesterday, a short, simple phrase yesterday follows the longer and more complex phrase that his cousins left, violating accessibility-based ordering. Alternative utterances that do follow accessibility include Yesterday, Tom said that his cousins left or Tom said yesterday that his cousins left. In these cases, however, the modifier obligatorily modifies said, eliminating these as options to convey the local modification left-yesterday meaning. Two alternatives are available that convey the local left-yesterday meaning. First, a producer could embed the yesterday within the sentential complement that his cousins left, yielding either that yesterday his cousins left or that his cousins yesterday left. These alternatives also do not follow accessibility; the 
long element, the embedded VP, begins before the short adverbial, and these orders may also be disfavored if the production system does not like to interrupt a message unit or constituent with another (Lohse, Hawkins, \& Wasow, 2004; Solomon \& Pearlmutter, 2004). Another alternative that conveys the left-yesterday meaning is for yesterday to follow left: Tom said that his cousins left yesterday, which as noted violates accessibility-based ordering. In sum, language producers have several accessibility-based word orders available to express the said-yesterday meaning, but not for the left-yesterday meaning. This means that, even though the verb1 . . verb2 modifier is technically ambiguous between distant (verb1) and local (verb2) modification interpretations, these production pressures should make it a much more common choice for expressing local modification, since better alternatives exist to convey distant interpretations. Consistent with this claim, Sturt, Costa, Lombardo, and Frasconi (2003) found 92\% local modifications in a sample of 380 verb modification ambiguities from the Wall Street Journal corpus.

Our claim then goes as follows: Phrase length and complexity affect accessibility of utterance components and, therefore, the order of these components in the sentences that producers say and write. These accessibility-based production patterns create a distributional pattern in the language, in which the technically ambiguous verb modifier construction is much more strongly associated with the local modification interpretation than with the distant modification interpretation. We hypothesize that language users implicitly learn this form-meaning mapping and apply this knowledge to subsequent sentence comprehension, so that when they encounter new input with verb modification ambiguities, they have a strong tendency to interpret them with the local modification interpretation, consistent with their past experience. Moreover, this tendency is relatively immune to changes in lexical content because the statistics of form-meaning co-occurrences are so skewed in the input and do not vary much with choice of lexical items. This tendency can be traced back to language production; the length differences between the embedded VP and the modifying phrase are often so large that this factor drives accessibility, with little effect of the particular lexical items in the utterance. Thus, in accord with this view, the local modification preference comes from comprehenders' learning and applying the statistics of the language, and the statistics of the language in turn come from accessibility-based production processes.

If language producers are responding to accessibility as described above, and if language comprehenders are learning the resulting statistical patterns of phrase length and interpretation in the language, then this account makes testable predictions for production and comprehension behavior when accessibility constraints are neutralized. For example, consider Mary likes swimming very much/slowly and Mary likes to swim very much/slowly, where very much modifies the distant verb likes and very slowly modifies the local verb swim(ming). In these cases, the embedded VPs swimming and to swim are only one or two words long and are, therefore, as short as or shorter than the modifier very much/slowly. In these cases, phrase length does not contribute much to word order choices, and any complexity differences to accessibility are also small, since complexity effects when length is controlled tend to be modest (Wasow \& Arnold, 2003). Thus, on accessibility grounds, there is little pressure to avoid the verb . . verb modifier construction during production planning, regardless of whether the intended interpretation is for the local modification, as in Mary likes swimming very slowly, or for the distant one, as in Mary likes swimming very much. If these hypotheses are correct, two predictions follow. First, utterances with short embedded verb phrases should frequently be uttered both when distant modification interpretations are intended and when local modification interpretations are intended, in contrast to utterances with long embedded verb phrases, which should skew heavily toward local modification interpretations. Second, if comprehenders have learned about form-meaning mappings that include information about phrase length, their interpretations should vary as a function of the length of the embedded VP. We tested the production predictions in two corpus analyses, examining whether modification ambiguities with a short embedded phrase tend to have a distant modification interpretation more often than those with a long embedded verb phrase. Following the corpus analyses, we tested the comprehension prediction in two self-paced reading studies that independently manipulated embedded phrase length and modification interpretation.

\section{Corpus Analyses}

The goal of the corpus analyses was to investigate the relationship between phrase length and interpretation in verb modification ambiguities. Since many corpora are too small to yield adequate numbers of verb modification ambiguities that have very short embedded verb phrases, we examined the role of phrase length using Google searches of Web pages, which have been shown to correlate well with more traditional corpus analyses (Keller \& Lapata, 2003).

We extracted 100 sentences in each of two searches according to the search criteria described below. The sentences were coded for distant versus local modification site by the authors and a linguistics student blind to the hypotheses of the study. Disagreements were rare $(<5 \%)$ and were resolved by reading and discussing the sentences in the broader context in which they appeared in the original Web page. Three items $(1.5 \%$, all with temporal modifications, such as very often) were ambiguous with regard to whether the distant or local verb was being modified; these were replaced by new sentences.

Search 1: Two-word embedded VPs. Our first goal was to examine interpretation preferences with the shortest embedded VPs that could be efficiently search fornamely, likes to verb very. . . structures. Given the view that local modification is almost universally favored in verb modification ambiguities, we first investigated whether there existed a construction with a very short embedded verb phrase that did not have a strong bias.

Preliminary use of Google search tools (the "advanced search" option on the Google home page) revealed that it would be necessary to specify some lexical items in the 
search string; more general searches did not successfully select for verb modification ambiguities. Three lexical items - likes, to, and very - were included in the first search.

Likes was specified as the distant verb because this verb frequently takes embedded verb phrases (e.g., likes to cook). Likes appears to have high modifiability and receives a reasonable number of modifications in verb modification ambiguities (e.g., likes to cook very much), so that this verb is a good choice for avoiding floor effects and observing any effects of length that might exist on modification patterns.

To was included to force the local verb to be an infinitive form, because generic gerund forms (likes Verbing) cannot be specifically searched for with Google search tools. Thus, all embedded verb phrases were infinitival complements of like.

Very was included to increase the rate of returning adverbial expressions (which necessarily modify verbs). Thus, all modifiers contained very.

We entered the string "likes to * very" into Google's search field; “*” was a wild card for any word or group of words. Sentences returned by this search were handfiltered until we found 100 tokens containing a single infinitive verb between to and very; examples include "... likes to play very much" and "likes to purr very loud for treats." In this set of 100 sentences, $41 \%$ contained distant modification, and 59\% had local modification, which is only a marginal bias in favor of the local modification interpretation, by binomial test of difference from $.5(.05<p<.10)$. This outcome is very different from the $92 \%$ local bias that Sturt et al. (2003) found with sentences of unrestricted phrase length and is consistent with the claim that length is a factor in the production of distant versus local modification sentences. We investigated this claim more systematically in Search 2.

Search 2: Embedded VPs with three or more words. We conducted searches for modification ambiguities with embedded infinitival expressions containing words before the infinitive (e.g., likes his mother to cook ...) and/or after the infinitive (to cook lasagna, to cook Mary some lasagna). We used the search strings "likes to * very," "liked to * very," and "like to * very" and hand-filtered the output until we found 100 sentences that matched our search criteria of an embedded infinitive verb with additional material either between the like-verb and the infinitive and/or material between the embedded verb and very. Examples include "I would have liked to talk to you very much," "The brain likes things to change very gradually," and "A lot of people in the town would have liked her just to go away very quietly."

The embedded verb phrases in the sentences ranged from 3 to 8 words, with a mean of 4.19 words. With these somewhat longer embedded verb phrases, 26 had distant modification and 74 had local modification. These values differed from the proportions found in the first search $\left(\chi^{2}=\right.$ $5.05, p<.05)$. These results suggest that length of the verb phrase influences distant versus local modification preferences. We investigated this hypothesis more precisely by analyzing the correlation between modification site and embedded verb phrase length in the sample: As embedded phrase length increased, the rate of local modification increased as well $[r=.19 ; F(98)=3.80, p=.05]$.

These results establish a foundation for the claim that phrase length is correlated with interpretation, and they set the stage for the behavioral experiments below. These results are also consistent with analyses of other constructions without verb modification ambiguities, which show that verbs and their modifiers tend to be close to each other in the verb phrase (see, e.g., Wasow \& Arnold, 2003). These patterns may similarly stem from accessibility and may also contribute to interpretations of verb modification ambiguities. Other, more complex corpus searches may prove to be important in investigating these possibilities; but at this point, a natural next step is to examine whether the length-based effects identified in the corpus influence online comprehension patterns. In Experiment 1, we used reading time measures to address the comprehension consequences of these different distributions of language use. If the interpretation patterns of modification ambiguities produced with short verb phrases are different from those of long verb phrases, and if comprehenders are guided by their experience with these patterns, they should interpret modification ambiguities differently, depending on the length of the verb phrase. This outcome would support the PDC account, whereas a uniform preference for local modification would be more consistent with claims for an inherent comprehension bias.

\section{EXPERIMENT 1}

As in the first corpus search, the goal for Experiment 1 was to examine whether shortest possible embedded VP still had a bias for local modification. More precisely, we investigated whether interpretation preferences, as measured by reading times, were reliably different in a long embedded VP, for which we expected a strong local modification preference, than in a short VP, for which we expected no preference.

\section{Method}

Participants. Seventy-three native-English-speaking undergraduates were paid $\$ 5$ for participation. Five participants were excluded from analyses either for less than $75 \%$ comprehension-question accuracy or for reading times more than $2 S D$ s above the grand mean for all participants on all items.

Materials and Design. Sentences were designed to cross two levels of embedded VP phrase length (short or long) and disambiguation (modifying the distant or local verb). The need to have a strong length manipulation and a clear disambiguation led us to choose gerund and infinitive phrases, such as swimming and to leave (see Table 1); all of the items are provided in Appendix A. These phrases have the advantage of being maximally short in the short condition, with the two verb phrases and disambiguation all being adjacent. Each sentence consisted of an initial NP (Mary, in the example), followed by two possible verb modification sites (e.g., likes and swimming), followed by a one- to four-word prepositional or adverbial phrase that plausibly modified either the distant or the local verb.

Four counterbalanced presentation lists were created, each containing 8 experimental and 61 filler sentences. The filler items comprised 40 items from two unrelated experiments and 21 sentences of varying structures.

Procedure. We used a single-word, self-paced reading task in which dashes initially indicated the location of sentence words on the screen (Just, Carpenter, \& Woolley, 1982). Participants pressed 
Table 1

Sample Materials From Experiment 1

\section{Short Condition}

Distant modification disambiguation: Mary likes swimming very much. Local modification disambiguation: Mary likes swimming very slowly.

$$
\text { Long Condition }
$$

Distant modification disambiguation: Mary likes it when the beautiful dolphins at Sea World are swimming very much.

Local modification disambiguation: Mary likes it when the beautiful dolphins at Sea World are swimming very slowly.

Note-The verb being modified and its modifier (the disambiguation phrase) are underlined.

the space bar to see each word of the sentence in a noncumulative fashion, and reading times formed the dependent measure. Sentences were followed by a yes/no comprehension question, which the participants answered with a keypress, and feedback on accuracy. Nine practice items were followed by one of the experimental presentation lists; the items appeared in random order.

\section{Results and Discussion}

Overall, participants answered $93.1 \%(S D=4.15)$ of the comprehension questions correctly, and no participant had an error rate greater than $20 \%$. Error rates on the comprehension questions did not vary significantly by condition. Items answered incorrectly were excluded from reading time analyses.

Prior to analysis, reading times greater than $2,500 \mathrm{msec}$ were removed $(<0.03 \%)$, and reading times were adjusted for word length (F. Ferreira \& Clifton, 1986) to remove effects of this factor. For the purpose of analysis, reading times were grouped together into two critical regions: the local verb (e.g., swimming) and the one- to four-word disambiguation phrase (e.g., very much/very slowly). This phrase was treated as a single region because the length varied across items and disambiguation was not always at the same point. Reading times more than $2 S D$ s from the cell mean for that region in each condition were Winsorized ( $<5 \%$ of observations).

The results are presented in Figure 1. ANOVAs were performed on each sentence region. In Region 1 (the verb), there was only a main effect of length $\left[F_{1}(1,67)=\right.$ 9.74, $\left.p<.005 ; F_{2}(1,7)=6.50, p<.05\right]$; reading times were shorter in the long condition than in the short condition. In Region 2 (the disambiguation), there was a reliable length $\times$ disambiguation interaction (marginal in the items analysis) $\left[F_{1}(1,67)=7.61, p<.01 ; F_{2}(1,7)=\right.$ $4.38, p=.075]$. The nature of this interaction was that, in the short condition, there was no difference between the distant and local modification interpretations $(F \mathrm{~s}<1)$; but in the long condition, reading times were significantly longer for the distant modification than for the local one $\left[F_{1}(1,67)=12.97, p<.001 ; F_{2}(1,7)=6.07, p<.05\right]$. This result is exactly as predicted, if phrase length indeed influences interpretations of modification ambiguity.

These results complement the first corpus analysis, showing that, when the embedded VP is an extremely short infinitive or gerund phrase, there is no difference in reading time between a local and a distant modification. Investigation of these very short embedded phrases was an important first step in demonstrating that the local modification bias is not universal across all tokens of this ambiguity and, in fact, is not present in these short phrase variants. One of the disadvantages of these very short phrases in an experiment, however, is that it is difficult to develop a large number of stimulus items without having excessive repetition in the stimulus set, since the shortness of the embedded phrase permits little variation across sentences. A second concern is that the short and long conditions differ in their syntactic structures, which could be another factor that shapes interpretation preferences. Experiment 2 addressed these concerns with a more subtle length manipulation that allowed both greater variety and a greater number of materials and also allowed the same syntactic structure to be used across short and long conditions.

\section{EXPERIMENT 2}

We used a more subtle manipulation of length in Experiment 2 than in Experiment 1 in order to examine length effects separately from effects of the syntactic structure

\section{Short Condition}

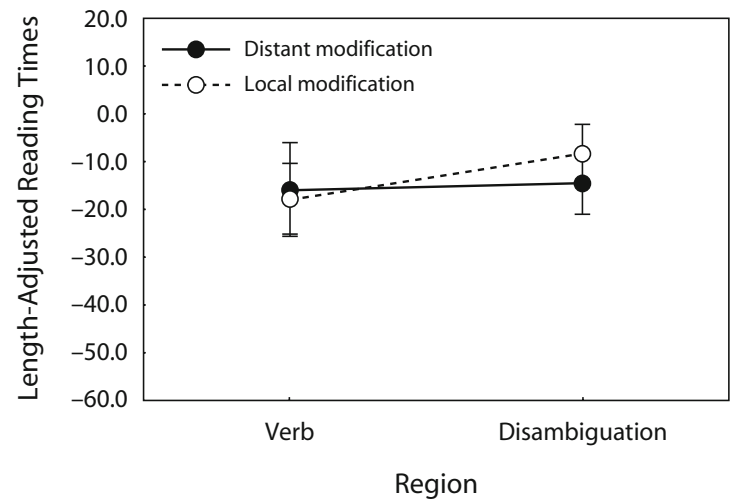

\section{Long Condition}

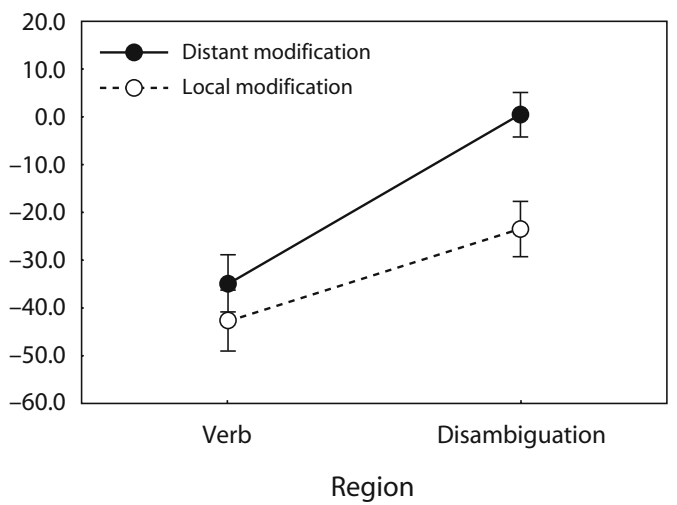

Figure 1. Mean length-adjusted reading times (with standard error bars), computed across participants, for both the distant and local modification disambiguation conditions from Experiment 1. 
Table 2

Sample Materials From Experiment 2

Short Condition

Distant modification disambiguation: One of the high points of this year's summer camp was when Mrs. Johnson taught the kids to dive in a single afternoon, and everyone had a lot of fun.

Local modification disambiguation: One of the high points of this year's summer camp was when Mrs. Johnson taught the kids to dive into the deep end, and everyone had a lot of fun.

\section{Long Condition}

Distant modification disambiguation: One of the high points of camp was when Mrs. Johnson taught the sixth and seventh grade kids to dive in a single afternoon, and everyone had a lot of fun.

Local modification disambiguation: One of the high points of camp was when Mrs. Johnson taught the sixth and seventh grade kids to dive into the deep end, and everyone had a lot of fun.

Note-The verb being modified and its modifier are underlined. In the short condition, there was a longer introductory phrase and a short distance between the main and the embedded verb. In the long condition, there was a shorter introductory phrase and a long distance between the main and the embedded verb.

of the embedded phrase. In Experiment 2, the items in the short condition were slightly longer than those in the short condition of Experiment 1; they resemble the tokens from Corpus Search 2 that had relatively short embedded phrases. The long condition in Experiment 2 contained embedded verb phrases that were analogous to the relatively long items found in Corpus Search 2. The corpus search yielded a correlation between length and rate of local modification. If the same pattern is observed in reading times, there should be a length $\times$ disambiguation interaction with little or no difference between local and distant modification in the short condition, but there should be longer reading times for distant modification than for local modification when the embedded VP is long.

\section{Method}

Participants and Procedure. Forty native-English-speaking undergraduates were paid $\$ 5$ for participation or received extra credit for an undergraduate psychology course. The procedure was identical to that in Experiment 1.

Materials and Design. The experimental materials consisted of 16 items crossing length and disambiguation factors (see Table 2); all of the items are provided in Appendix B. Sentences began with an introductory phrase or clause followed by the ambiguity and disambiguation and ending with a final clause. Each sentence had a complex verb phrase containing a noun phrase that separated the

\section{Short Condition}

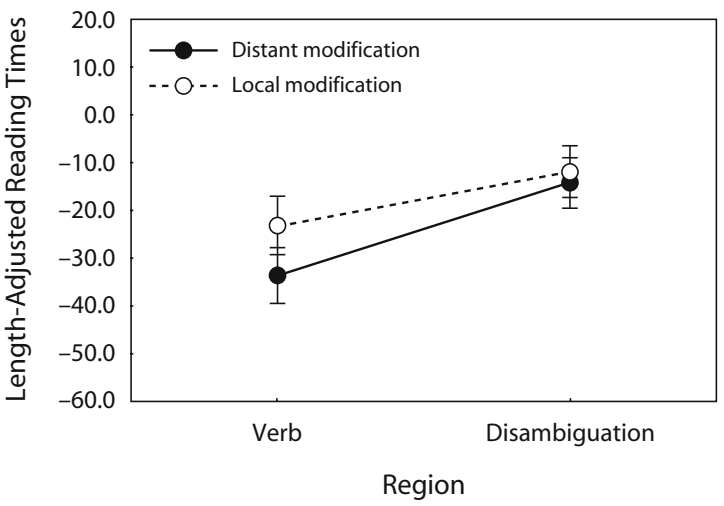

two verbs (e.g., the kids in taught the kids to dive). Following the embedded verb was a disambiguating prepositional or adverbial phrase that modified either the distant verb (e.g., in a single afternoon, modifying taught) or the local (embedded) verb (into the deep end, modifying dive). The length of the noun phrase between the two verbs varied: The verbs were separated by one or two words in the short condition and by five to eight words in the long condition. To balance the overall sentence length in the two length conditions, the introductory phrase length was varied, so that the overall sentence length varied across conditions by no more than two words.

\section{Results and Discussion}

The participants answered $90.1 \%(S D=7.80)$ of the comprehension questions correctly. Error rates did not vary significantly by condition. Items answered incorrectly were excluded from reading time analyses. Reading times were length adjusted, grouped into regions, and trimmed, as in Experiment 1. Trimming affected less than $5 \%$ of the data. Reading times are shown in Figure 2. In Region 1 (the local verb), reading times were somewhat shorter in the short condition than in the long condition $\left[F_{1}(1,39)=6.64, p<.05 ; F_{2}(1,15)=1.84\right.$, n.s.]. In Region 2 (the disambiguation), there was again a significant length $\times$ disambiguation interaction $\left[F_{1}(1,39)=8.25\right.$, $\left.p<.01 ; F_{2}(1,15)=4.63, p<.05\right]$, so that, in the short condition, there was no difference between the distant and

Figure 2. Mean length-adjusted reading times (with standard error bars), computed across participants, for both the distant and local modification disambiguation conditions from Experiment 2. 
local modifications $\left(F_{\mathrm{s}}<1\right)$, whereas in the long condition, the reading times for the distant modification were significantly longer than those for the local modification $\left[F_{1}(1,39)=12.30, p<.001 ; F_{2}(1,15)=7.42, p<.05\right]$. These results replicated the effects of phrase length on interpretation preferences found in Experiment 1, while controlling syntactic structure across short and long conditions, so that only the length of a noun phrase varied. The results confirm the patterns shown in Corpus Search 2, in which increasing length was correlated with increasing the rate of local modification interpretation.

\section{GENERAL DISCUSSION}

Both the corpus analyses and the experiments presented here demonstrate that phrase length has a robust effect on the processing of modification ambiguities. In both experiments, a local modification preference was observed only when the potential modification sites were separated by five or more words. When the sites were adjacent, as in Experiment 1, or separated by a short noun phrase, as in Experiment 2, no local modification preference was observed. Similarly, in the corpus searches, sentences with few words in the embedded verb phrase were more likely to have a distant modification interpretation than those with longer embedded verb phrases.

These results are important in several ways. First, they show a relationship between the distributional patterns in the input and online comprehension. To the extent that such relationships continue to be demonstrated, these results support constraint-based accounts of comprehension over ones that suggest that comprehension is not driven by the frequency of alternative interpretations (Gibson \& Schütze, 1999; but see Desmet \& Gibson, 2003). Second, these results suggest that the verb modification ambiguity is not overwhelmingly biased to local modification, as might be assumed from the literature; rather, the extent to which local modification is preferred is a function of the length of the embedded phrase. Most investigations of this ambiguity have tended to use embedded phrases longer than those used in the short conditions in the present study.

Third, these results support the claims of the PDC account linking comprehension behavior to pressures on language production (Gennari \& MacDonald, 2009; MacDonald, 1999). In the case of modification ambiguities, we suggest that accessibility-based production processes modulate the correlation between form and meaning in verb modification ambiguities and that comprehenders implicitly learn these patterns and apply them to interpretations of new input. As a result, comprehension patterns for short items show no interpretation preference (reflecting the lack of a strong bias in previously encountered sentences), but there is a clear local modification bias for long items, for which there is a strong asymmetry in past input. The present length-based results and the claim for a learning-based account of the interpretation preferences constitute a marked departure from the assumption that modification ambiguities are initially interpreted to have local modification, owing to inherent biases in the comprehension architecture (e.g., Frazier, 1987; Kimball, 1973). Our results also support the novel notion that comprehension behavior can be traced not to an architectural feature of the comprehension system but to architectural features of the language production system - in this case, language producers' tendencies to be guided by the relative accessibility of phrases during production planning.

In the following sections, we consider the implications of these results in several respects. First, we return to an issue raised in the introduction - namely, the nature of the statistical learning that is hypothesized to drive the lengthbased interpretation preferences within constraint-based accounts and the PDC. Second, we place these results in the context of other studies of the effects of phrase length on interpretation preferences.

\section{Lexical Versus Nonlexical Statistics}

As we noted in the introduction, the effects of lexical items have been thought to be relatively mild in verb modification ambiguities. This state of affairs seems rather different from that with other syntactic ambiguities, but this impression may turn out to be false. Other ambiguities also once seemed impervious to lexical properties; for example, it took about a decade for researchers to identify the main lexical and discourse constraints that seem to influence the interpretation of main verb-reduced relative ambiguities, in contrast to the then-dominant view that interpretation was shaped by innate parsing principles (MacDonald \& Seidenberg, 2006). There are clear cases in which lexical items can affect interpretation of verb modification ambiguities (Fodor \& Inoue, 1994), and from the present data, we now know that there are other factors (e.g., length) that can reduce the strong bias for one interpretation. This situation suggests that it may be possible to use relatively unbiased sentences to study the role of lexical constraints further.

More broadly, the data from the present experiments and corpus analyses suggest that comprehenders are learning distributional patterns that need not be tied to particular words. Even though constraint-based accounts have emphasized lexical learning, learning about more abstract patterns is possible, perhaps as a generalization over individual words. This level of learning may underlie biases toward more common sentence structures independent of lexical items (as in the main-verb/reduced relative ambiguity). What the PDC account can add is an explanation of why certain abstract patterns exist; in the case of verb modification ambiguities, they stem from accessibility-based production processes.

\section{Length Effects in Comprehension}

Our demonstration of phrase length effects in verb modification ambiguities complements several studies that have found length effects in other ambiguous constructions (e.g., F. Ferreira \& Henderson, 1991; Fodor, 1998; Gibson, 1998; Thornton, MacDonald, \& Arnold, 2000; Warner \& Glass, 1987). Pynte (2006) manipulated constituent length as a means of varying prosody of several ambiguous and unambiguous modifier constructions in French and found some effects of length in these cases, which he interpreted as evidence for Fodor's same-sizesister principle, in which comprehenders tend to expect 
constituents of similar length. Most similar to our studies, Carlson, Clifton, and Frazier (2001) investigated the effect of phrase length on verb modification ambiguities; but rather than manipulate the length of the embedded VP, as we have done, they manipulated the length of the modifying expression, with items such as "Susie learned that Bill telephoned [extremely late] last night" (with the material in brackets being present in a long modifier condition and absent in a short modifier condition). They found no effects of this length manipulation in either reading times or in the final interpretations that participants adopted. This finding is consistent with our data, in that Carlson et al.'s materials were very similar to the short condition in our Experiment 2, with two words separating the potential modification sites; we also found no reading time difference between local and distant modification.

Carlson et al. (2001) noted that their data were inconsistent with another length-based account, Fodor's (1998) same-size-sister principle, which explicitly predicts more distant modification for Carlson et al.'s long modifier conditions. Additionally, our approach differs from Fodor's in suggesting that the effects emerge from experience with the language, rather than from an inherent bias in the human comprehension system. Demonstrations of length effects in comprehension cannot by themselves indicate whether they stem from a hardwired feature of the comprehension system or emerge from learning the production-based distributional regularities in the language. In the PDC's favor, it is already well established that phrase length does affect ordering in production (De Smedt, 1994; Stallings et al., 1998). The account also builds on extensive work within constraint-based sentence processing that shows that comprehenders weigh probabilistic information about the likelihood of alternative interpretations of ambiguities (for a review, see MacDonald \& Seidenberg, 2006). The account also builds on work showing that humans are powerful statistical learners of linguistic information (Saffran, 2003), including learning of distributional information relevant to ambiguity resolution in sentence processing (Wells et al., 2009). The PDC approach therefore integrates already-established claims in production, learning, and comprehension to provide a novel explanation for an old result - that is, that people strongly prefer to interpret sentences like "Tom said that his cousins left yesterday" with local modification, so that yesterday modifies the leaving event, not the saying event. Moreover, PDC is an explicit model of the relationship between production and comprehension, a topic of increasing importance in psycholinguistics (see, e.g., Chang, Dell, \& Bock, 2006; Pickering \& Garrod, 2004).

It is possible that future research will be able to reconcile the PDC approach with the tenets of at least some of the comprehension-specific approaches. For example, Fodor's (1998) argument for a greater role for prosodic information in interpretation is not inconsistent with constraint-based accounts, even if the specifics of the same-size-sister principle prove incorrect. Similarly, Gibson (1998) proposed that there are memory and integration costs associated with various dependencies in a sentence, and computational limitations on comprehension are also consistent with constraint-based accounts (Gennari \& MacDonald, 2008,
2009). In Gibson's account, costs increase as the distance between dependent elements in a sentence increases. It is possible that the PDC approach and Gibson's account are not mutually exclusive - that is, that there are length-based constraints that shape both production and comprehension processes. Alternatively, the length-based constraints that Gibson has formalized for comprehension may instead reflect constraints in production; perhaps his memory-based account is a fair approximation of the memory demands that producers experience. In this view, Gibson's locality account could offer a perspective that is somewhat different from the accessibility claims in the production literature (e.g., De Smedt, 1994). If so, locality would be a force that drives production patterns, creating distributional patterns in the input that are learned by comprehenders. In this view, comprehenders' behavior would be captured by a locality metric not because locality is an inherent part of the parsing architecture, but because locality guides production patterns, creating distributional patterns that comprehenders learn and apply to new input. Again, a demonstration of an effect in comprehension by itself does not indicate whether the effect owes to hardwired comprehension processes or to the learning of statistical patterns in the input and their application during constraint-based sentence processing. It is likely that learning studies and computational models (as in Wells et al., 2009) will be highly informative about these alternative perspectives.

\section{AUTHOR NOTE}

This research was supported by NSF Grant SBR-9511270 and NIH Grants R01HD47425 and K01AG025195. Correspondence concerning this article should be addressed to M. C. MacDonald, Department of Psychology, 1202 W. Johnson St., University of Wisconsin-Madison, Madison, WI 53706 (e-mail: mcmacdonald@wisc.edu).

\section{REFERENCES}

Altmann, G. T. M., van Nice, K. Y., Garnham, A., \& Henstra, J.-A. (1998). Late closure in context. Journal of Memory \& Language, $\mathbf{3 8}$, 459-484.

Bock, K. (1987). An effect of the accessibility of word forms on sentence structure. Journal of Memory \& Language, 26, 119-137.

Carlson, K., Clifton, C., JR., \& Frazier, L. (2001). Prosodic boundaries in adjunct attachment. Journal of Memory \& Language, 45, 5881. doi:10.1006/jmla.2000.2762

Chang, F., Dell, G. S., \& Bock, J. K. (2006). Becoming syntactic. Psychological Review, 113, 234-272. doi:10.1037/0033-295X.113.2.234

DE SmedT, K. J. M. J. (1994). Parallelism in incremental sentence generation. In G. Adriaens \& U. Hahn (Eds.), Parallel natural language processing (pp. 421-447). Norwood, NJ: Ablex.

Desmet, T., \& GiBson, E. (2003). Disambiguation preferences and corpus frequencies in noun phrase conjunction. Journal of Memory \& Language, 49, 353-374. doi:10.1016/S0749-596X(03)00025-1

FERrEIRA, F. (1991). Effects of length and syntactic complexity on initiation times for prepared utterances. Journal of Memory \& Language, 30, 210-233.

Ferreira, F., \& Clifton, C., JR. (1986). The independence of syntactic processing. Journal of Memory \& Language, 25, 348-368.

FERreira, F., \& Henderson, J. M. (1991). Recovery from misanalyses of garden-path sentences. Journal of Memory \& Language, 30, 725745

Ferreira, V. S. (1996). Is it better to give than to donate? Syntactic flexibility in language production. Journal of Memory \& Language, 35, 724-755.

FoDOR, J. D. (1998). Learning to parse? Journal of Psycholinguistic Research, 27, 285-319. 
Fodor, J. D., \& Inoue, A. (1994). The diagnosis and cure of garden paths. Journal of Psycholinguistic Research, 23, 407-434.

Frazier, L. (1987). Sentence processing: A tutorial review. In M. Coltheart (Ed.), Attention and performance XII: The psychology of reading (pp. 559-586). Hillsdale, NJ: Erlbaum.

Gennari, S. P., \& MacDonald, M. C. (2008). Semantic indeterminacy in object relative clauses. Journal of Memory \& Language, 58, 161187. doi:10.1016/j.jml.2007.07.004

Gennari, S. P., \& MacDonald, M. C. (2009). Linking production and comprehension processes: The case of relative clauses. Cognition, 111, 1-23. doi:10.1016/j.cognition.2008.12.006

GiBson, E. (1998). Linguistic complexity: Locality of syntactic dependencies. Cognition, 68, 1-76.

Gibson, E., \& SchüTZE, C. T. (1999). Disambiguation preferences in noun phrase conjunction do not mirror corpus frequency. Journal of Memory \& Language, 40, 263-279.

HaWkIns, J. A. (1994). A performance theory of order and constituency. New York: Cambridge University Press.

Just, M. A., Carpenter, P. A., \& Woolley, J. D. (1982). Paradigms and processes in reading comprehension. Journal of Experimental Psychology: General, 111, 228-238.

Keller, F., \& Lapata, M. (2003). Using the Web to obtain frequencies for unseen bigrams. Computational Linguistics, 29, 459-484.

Kempen, G., \& HoenKamp, E. (1987). An incremental procedural grammar for sentence formulation. Cognitive Science, 11, 201-258.

Kimball, J. (1973). Seven principles of surface structure parsing in natural language. Cognition, 2, 15-47.

Lohse, B., Hawkins, J. A., \& Wasow, T. (2004). Domain minimization in English verb-particle constructions. Language, 80, 238-261.

MaCDonald, M. C. (1999). Distributional information in language comprehension, production, and acquisition: Three puzzles and a moral. In B. MacWhinney (Ed.), The emergence of language (pp. 177-196). Mahwah, NJ: Erlbaum.

MacDonald, M. C., \& Seidenberg, M. S. (2006). Constraint satisfaction accounts of lexical and sentence comprehension. In M. J. Traxler \& M. A. Gernsbacher (Eds.), Handbook of psycholinguistics (2nd ed., pp. 581-612). San Diego: Academic Press.

Pearlmutter, N. J., \& Gibson, E. (2001). Recency in verb phrase attachment. Journal of Experimental Psychology: Learning, Memory, \& Cognition, 27, 574-590. doi:10.1037/0278-7393.27.2.574

Pickering, M. J., \& GARRod, S. (2004). Toward a mechanistic psychology of dialogue. Behavioral \& Brain Sciences, 27, 169-226.

Pynte, J. (2006). Phrasing effects in comprehending PP constructions. Journal of Psycholinguistic Research, 35, 245-265. doi:10.1007/ s10936-006-9014-y

Race, D. S., \& MacDonald, M. C. (2003). The use of "that" in the production and comprehension of object relative clauses. In R. Alterman \& D. Hirsh (Eds.), Proceedings of the 25th Annual Conference of the Cognitive Science Society (pp. 946-951). Austin, TX: Cognitive Science Society.

SAFFran, J. R. (2003). Statistical language learning: Mechanisms and constraints. Current Directions in Psychological Science, 12, 110114

Solomon, E. S., \& Pearlmutter, N. J. (2004). Semantic integration and syntactic planning in language production. Cognitive Psychology, 49, 1-46. doi:10.1016/j.cogpsych.2003.10.001

Spivey-Knowlton, M., \& Sedivy, J. C. (1995). Resolving attachment ambiguities with multiple constraints. Cognition, 55, 227-267.

Stallings, L. M., MacDonald, M. C., \& O'Seaghdha, P. G. (1998). Phrasal ordering constraints in sentence production: Phrase length and verb disposition in heavy-NP shift. Journal of Memory \& Language, 39, 392-417.

Sturt, P., Costa, F., Lombardo, V., \& Frasconi, P. (2003). Learning first-pass structural attachment preferences with dynamic grammars and recursive neural networks. Cognition, 88, 133-169. doi:10.1016/ S0010-0277(03)00026-X

Tanenhaus, M. K., \& Trueswell, J. C. (1995). Sentence comprehension. In J. L. Miller \& P. D. Eimas (Eds.), Handbook of perception and cognition: Vol. 11. Speech, language, and communication (pp. 217262). San Diego: Academic Press.

Thornton, R., MacDonald, M. C., \& Arnold, J. E. (2000). The concomitant effects of phrase length and informational content in sentence comprehension. Journal of Psycholinguistic Research, 29, 195-203.

Thornton, R., MacDonald, M. C., \& Gil, M. (1999). Pragmatic constraint on the interpretation of complex noun phrases in Spanish and English. Journal of Experimental Psychology: Learning, Memory, \& Cognition, 25, 1347-1365.

Trueswell, J. C. (1996). The role of lexical frequency in syntactic ambiguity resolution. Journal of Memory \& Language, 35, 566-585.

Warner, J., \& Glass, A. L. (1987). Context and distance-todisambiguation effects in ambiguity resolution: Evidence from grammaticality judgments of garden path sentences. Journal of Memory \& Language, 26, 714-738.

WASOW, T. (1997). Remarks on grammatical weight. Language Variation \& Change, 9, 81-105.

WASOW, T., \& ARNold, J. (2003). Post-verbal constituent ordering in English. In G. Rohdenburg \& B. Mondorf (Eds.), Determinants of grammatical variation in English (pp. 119-154). Berlin: Walter de Gruyter.

Wells, J. B., Christiansen, M. H., Race, D. S., Acheson, D. J., \& MacDonald, M. C. (2009). Experience and sentence processing: Statistical learning and relative clause comprehension. Cognitive Psychology, 58, 250-271. doi:10.1016/j.cogpsych.2008.08.002

WhEELDON, L., \& LAHIRI, A. (1997). Prosodic units in speech production. Journal of Memory \& Language, 37, 356-381.

\section{APPENDIXA
Stimuli Used in Experiment 1}

Short conditions were changed to long conditions by adding the bracketed material marked with a plus sign ("+"). Disambiguations begin after the double slash, where slight word changes across length conditions are indicated in brackets. Final clauses, if any, are separated by a comma.

1. After the battle, Captain Hadley wanted [+ the two Russian generals in the prison] to die //with all his heart/with \{his/their\} boots on.

2. Mary likes [+it when the beautiful dolphins at Sea World are] swimming //very much/very slowly.

3. Although her sister isn't interested, Kimberly likes [+it when the Olympic athletes on TV are] skating //very much/very slowly.

4. Susan loves [+for her two teenage daughters] to jog //more than $\{$ long: + for them $\}$ to skate/more than five miles every day.

5. Fred promised [+his elderly uncle and aunt] to leave //repeatedly/quietly, but he always broke his word.

6. Kathy explained [+the importance of] gardening //patiently/using natural fertilizer, but no one was paying very much attention.

7. Jerry vowed [ + to his doctor] to diet //in a solemn voice/for three whole months, but the very next day he ate three Twinkies.

8. After two hours at the boring party, David wanted [+ those stupid talkative Harris twins] to leave //with all his heart/with the other guys, but his cousin forced him to stay. 


\section{APPENDIX B}

\section{Stimuli Used in Experiment 2}

Short conditions were changed to long conditions by removing words marked with a minus sign ("-_) from the introductory phrase, unless otherwise noted, and adding bracketed material marked with a plus sign ("+"). Disambiguations begin at the double slash and are given in the order distant/local, and final clauses follow the comma.

1. It wasn't until [-fairly early last] Tuesday afternoon that I explained my [+strange and oftentimes overwhelming] need to garden //to my next-door neighbor/with a new fertilizer, and I think I sounded a bit crazy.

2. Sue had always told [-just about] anyone who would listen that she loves for her [+lazy and overweight teenage] son to jog //more than for him to sleep/more than two miles a day, and that she bought him new running shoes.

3. It was [-around four o'clock] last Saturday afternoon when Tim spied [+ his eleventh grade Chemistry teacher] Mrs. Jones sunbathing//through the curtains/in her bikini, but she didn't notice him.

4. He had only been home for about an hour [-and a half] when Richard saw his wife [+ and a number of her coworkers] leave //with his own eyes/in his new truck, even though she had an accident earlier that week.

5. Because [-many] of the [-employees ignored the recent] schedule change, the boss reminded Ann [ + and a number of her co-workers] to leave //in a condescending voice/for the important meeting, but she didn't leave until much later.

6. It had been more than fifteen [ - and a half] years since Vera witnessed the [+ insane and extremely violent] criminal stealing //with her own eyes/from the cash register, so her testimony was discounted.

7. Last night, during the [-bottom of the] seventh inning, the catcher wanted the [+obnoxious loudmouthed] opposing batter to strike out//very much/very quickly, but he hit a home run!

8. Because the kitchen was [ + messy] [ - a terrible mess] after the remodeling, Mrs. Brown motivated her son [+and some of his fraternity brothers] to clean //by making cookies/with scouring pads, and he/ they had the place clean in no time.

9. During the [-town's annual Cinco de Mayo] festival, Elizabeth asked [ +her lively and outgoing friend] Eric to dance //in a meek voice/with her older sister, but just then the music stopped.

10. Since everyone [short: was really enjoying that summer's church talent show/long: enjoyed the show], the audience persuaded Tina [ + and the rest of the choir] to sing //with cheers and applause/for another twenty minutes, and she loved it.

11. After [-several] months [short: of trying to decide/long: deciding] exactly what to do, Mrs. Davis stopped her [+ amazingly wild and outrageous son] son from dating //by reducing his allowance/all through the weekend, but he found other ways to go out.

12. One of the high points of [-this year's summer] camp was when Mrs. Johnson taught the [+ sixth and seventh grade] kids to dive //in a single afternoon/into the deep end, and everyone had a lot of fun.

13. Just before her [ - final] chemistry test [ - of the semester], Jenny begged [ + her annoying and overbearing brother] Paul to help her//in a pleading voice/with the periodic table, but he refused.

14. It was just after [ - the high school's] graduation [- ceremony] that Mrs. Woo encouraged [+ her very favorite grandson] Neal to succeed //with her brilliant speech/with his college studies, and he was forever grateful.

15. Because the family was very poor [-and had little education], Aunt Sally wanted [+her unmotivated and extremely lazy] nephew Jim to graduate //with all her heart/with a college degree, but he dropped out instead.

16. It was just after the [-big] stock market crash [-last year] when the bank forced the [+very poor and underprivileged] tenants to move //by suing them all/by the month's end, so everyone had to find a new apartment.

(Manuscript received October 3, 2008;

revision accepted for publication May 31, 2009.) 